Figure 1. Clinical outcomes in ABA- vs ADA-treated pts at Wk 24 by SE genotype: as-treated analysis population

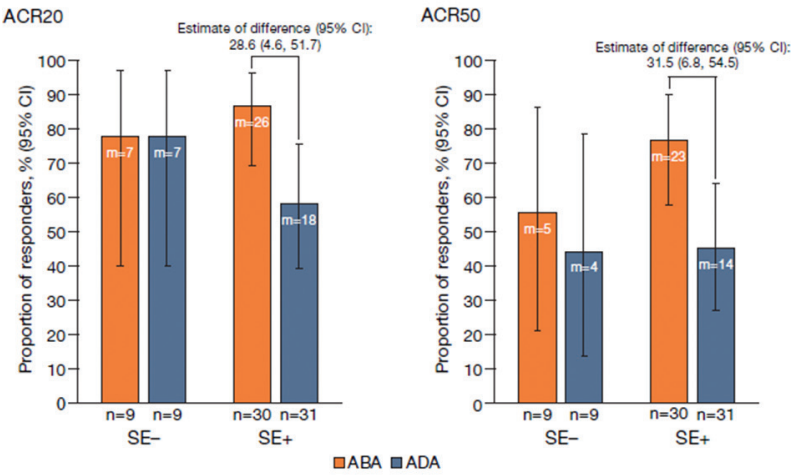

ACR70

DAS28 (CRP) remission
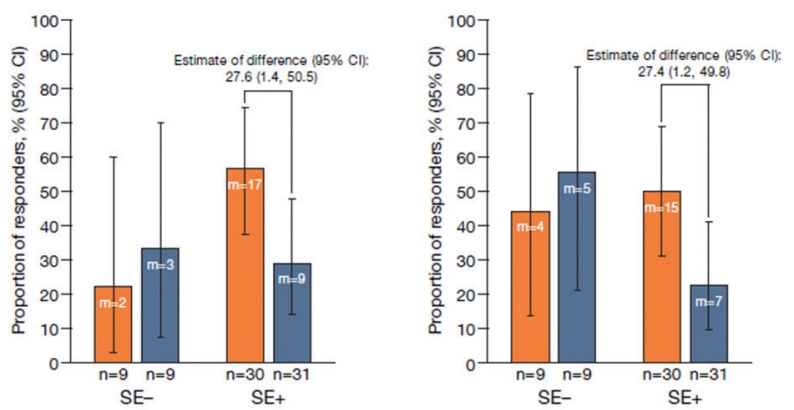

$m$ indicates the number of patients with a response; $n$ indicates the number of patients in the group. Pationts with missing data for efficacy parameters at Week 24 are imputed as non-responders

ADA=adalimumab; SE=shared epitope

study in which greater efficacy was seen with abatacept, but not TNF inhibitors, in anti-CCP2+ vs anti-CCP2- pts with RA (Harrold et al. J Rheumatol 2018).

Acknowledgement: Marianne Peluso (protocol manager); medical writing: Lola Parfitt (Caudex; funding: BMS)

Disclosure of Interests: William Rigby Consultant for: AbbVie, BMS, Genentech and Pfizer, Jane Buckner Grant/research support from: Janssen, BristolMyers Squibb (current); Novo Nordisk, Pfizer, Eli Lilly (past), Consultant for: Bristol-Myers Squibb, Eli Lilly, Lou Bridges: None declared, Marleen Nys Shareholder of: Bristol-Myers Squibb, Employee of: Bristol-Myers Squibb, Sheng Gao Shareholder of: Bristol-Myers Squibb, Employee of: Bristol-Myers Squibb, Martin Polinsky Shareholder of: Bristol-Myers Squibb, Employee of: Bristol-Myers Squibb, Alyssa Johnsen Shareholder of: Bristol-Myers Squibb, Employee of: Bristol-Myers Squibb, Neelanjana Ray Shareholder of: Bristol-Myers Squibb, Employee of: Bristol-Myers Squibb, Vivian Bykerk Grant/research support from: Mallinckrodt, BMS, Crescendo Biosciences, Sanofi/Regeneron., Consultant for: Amgen, Pfizer, UCB, Scipher, Sanofi/Genzyme/Regeneron

DOI: 10.1136/annrheumdis-2019-eular.8668

\section{LB0009 FIRST-IN-HUMAN STUDY OF NOVEL IMPLANTED VAGUS NERVE STIMULATION DEVICE TO TREAT RHEUMATOID ARTHRITIS}

Mark C. Genovese ${ }^{1}$, Norman Gaylis ${ }^{2}$, David Sikes $^{3}$, Alan Kivitz ${ }^{4}$, Diane M Horowitz ${ }^{5}$, Charles Peterfy ${ }^{6}$, Yaakov Levine ${ }^{7}$, David Chernoff ${ }^{7}$. ${ }^{1}$ Stanford University School of Medicine, Stanford, United States of America; ${ }^{2}$ Arthritis and Rheumatic Disease Specialties, Aventura, United States of America; ${ }^{3}$ Florida Medical Clinic, Zephyrhills, United States of America; ${ }^{4}$ Altoona Center for Clinical Research, Duncansville, United States of America; ${ }^{5}$ Northwell Health, Great Neck, United States of America; ${ }^{6}$ Spire Sciences, Boca Raton, United States of America; ${ }^{7}$ SetPoint Medical, Valencia, United States of America

Background: The inflammatory reflex plays a role in regulating innate and adaptive immunity through cellular and molecular pathways ${ }^{1}$. Activation of this neuroimmune reflex by electrical vagus nerve stimulation (VNS) reduced systemic inflammation and improved disease activity in a 17 subject rheumatoid arthritis (RA) proof-of-concept study using a reprogrammed epilepsy stimulator ${ }^{2}$. A novel miniaturized neurostimulator, the "MicroRegulator" (MR), was developed for a first-in-human pilot study in multi-drug refractory RA.

Objectives: To assess the safety and efficacy of the MR in a double-blind study in active RA patients.
Methods: The MR was implanted in 14 patients with active RA and prior insufficient response to $=2$ bDMARDS or JAK inhibitors with $=2$ different modes of action; all patients remained on stable background of methotrexate. Three weeks after implantation, the first 3 subjects were stimulated 1 min QD and, following safety review board approval, the remaining 11 patients were implanted with the MR and randomized to $1 \mathrm{~min}$ of sham, QD, or QID stimulations for 12 weeks. Patients, rheumatologists, joint assessors and monitors were fully blinded to treatment arm. Subjects randomized to sham had their devices activated after the primary endpoint at 12 weeks. Clinical efficacy was measured by DAS28-CRP response and contrast-enhanced MRI (RAMRIS OMERACT). The pharmacodynamic response to VNS was assessed in blood using cytokine production in an ex-vivo bioassay (TruCulture).

Results: 14 patients were enrolled (mean prior bDMARDs $=4.8$, mean DAS28$\mathrm{CRP}=5.94$ ). Implantation and stimulation were generally well tolerated. There were no device or treatment-related SAEs and 2 surgery related adverse events (left vocal cord paralysis, Horner's syndrome) that resolved without clinically significant sequelae. DAS28-CRP change at week 12 was (mean \pm SEM): Open label $\mathrm{QD}=-1.44 \pm 0.64, \mathrm{QD}=-1.24 \pm 0.88, \mathrm{QID}=0.38 \pm 0.71$, Sham $=0.16 \pm 0.21$ Of QD stimulated patients, 4 of 6 had a EULAR good or moderate response. MRI measures of synovitis or osteitis did not change after 12 weeks of stimulation. RAMRIS erosion scores correlated with EULAR response (change \pm SEM in ero sion scores in EULAR responders = $-2.2 \pm 1.4$ vs. $2.4 \pm 0.96$ in EULAR non-res ponders). The pharmacodynamic response to VNS was confirmed in actively stimulated groups with $>30 \%$ decrease from baseline in bioassay levels of IL- $1 \beta$, IL-6, and TNF-a at week 12.

Conclusion: The novel MR device and stimulation was well tolerated independent of the two surgery-related events. MR associated stimulation reduced signs and symptoms of RA in a meaningful number of highly drug-refractory subjects. No clinical improvement was observed in the sham group. These initial pilot data support the use of the MR in a larger blinded sham-controlled study in patients who have failed biologics or targeted oral therapies as a novel approach for treatment of RA and other chronic inflammatory diseases.

\section{REFERENCES}

[1] Andersson U, Tracey K, Annu. Rev. Immunol. 2012; 30:313.

[2] Koopman, F, et. al. PNAS. 2016; 113(29):8284

Disclosure of Interests: Mark C. Genovese Grant/research support from: Sanofi/Genzyme, Genentech/Roche, RPharm, Consultant for: Sanofi/Genzyme, Genentech/Roche, RPharm, Norman Gaylis Grant/research support from: Multiple clinical research trials, BMS, AbbVie, GSK, Janssen, Amgen, Pfizer, Regeneron, UCB, Sanofi, SetPoint, ImmunPharma, Astra Zeneca, Sandoz, Novartis, Gilead, Consultant for: electroCore, David Sikes Grant/research support from: SetPoint MedicalPfizerActelionAbbvieEli Lilly, Alan Kivitz Shareholder of: Novartis, Consultant for: Abbvie, Janssen, Pfizer, UCB, Genzyme, Sanofi, Regeneron, Boehringer Ingelheim, Sun Pharma Advanced Research, Flexion., Paid instructor for: Celgene, Horizon, Merck, Novartis, Pfizer, Genzyme, Sanofi, Regeneron Speakers bureau: Celgene, Horizon, Merck and Genetech, Flexion, Diane M Horowitz Grant/research support from: SetPoint Medical, Charles Peterfy Shareholder of: Spire Sciences, Inc, Consultant for: AbbVie, Acerta, Amgen, AstraZeneca, Bris tol-Myers Squibb, Centrexion, Daiichi Sankyu, Five Prime Therapeutics, Genentech, Hoffmann-La Roche, Janssen, Lilly USA, Medlmmune, Merck, Novartis Plexxikon, Pfizer, Sanofi, Salix-Santarus, Samsung, Employee of: Spire Sciences, Inc, Speakers bureau: Amgen, Yaakov Levine Shareholder of: SetPoint Medical Employee of: SetPoint Medical, David Chernoff Shareholder of: SetPoint Medical, Consultant for: Crescendo BioScience, Employee of: SetPoint Medical DOI: 10.1136/annrheumdis-2019-eular.8716

\section{LB0010 ULTRA-LOW DOSES OF RITUXIMAB OR RETREATMENT OF RHEUMATOID ARTHRITIS: A RANDOMISED CONTROLLED NON-INFERIORITY TRIAL}

L.M. Verhoef ${ }^{1}$, Nathan den Broeder ${ }^{2}$, R.M. Thurlings ${ }^{3}$, W.H. van der Laan ${ }^{4}$, W. van der Weele ${ }^{5}$, Marc Kok ${ }^{5}$, H.J. Bernelot Moens ${ }^{5}$, Thasia Woodworth ${ }^{5}$, Bart van den Bemt ${ }^{6}$, Frank van den Hoogen ${ }^{5}$, Alfons den Broeder ${ }^{5} .{ }^{1}$ Sint Maartenskliniek, Rheumatology, Nijmegen, Netherlands; ${ }^{2}$ Radboudumc, Rheumatology, Nijmegen, Netherlands; ${ }^{3}$ Reade, Rheumatology, Amsterdam, Netherlands; ${ }^{4}$ Maasstad hospital, Department of Rheumatology and Clinical Immunology, Rotterdam, Netherlands; ${ }^{5}$ Ziekenhuisgroep Twente, Rheumatology, Almelo, Netherlands; ${ }^{6}$ David Geffen School of Medicine, University of California, Division of Rheumatology, Los Angeles, United States of America; ${ }^{7}$ Sint Maartenskliniek, Pharmacy, Nijmegen, Netherlands; ${ }^{8}$ Radboudumc, Pharmacy, Nijmegen, Netherlands

Background: Rituximab (RTX) is an effective treatment for patients with Rheu matoid Arthritis $(R A) .1000 \mathrm{mg}(1 \times 1000 \mathrm{mg}$ or $2 \times 500 \mathrm{mg})$ has similar 6 -month efficacy as the registered dose of $2 \times 1000 \mathrm{mg}$. Based on several case reports and a 